\title{
A mysterious clavicular swelling
}

\author{
Nur Saadah Mohamad, Mohd Jazman Che Rahim, Azliana Aziz \\ Mohamad NS, Che Rahim MJ, Aziz A. A mysterious clavicular swelling. Malays Fam Physician. 2021;16(3);97-100. https://doi.org/10.51866/cr1113
}

\section{Keywords:}

Clavicular metastasis, non-

small cell lung carcinoma,

clavicular mass, lung

adenocarcinoma

\section{Authors:}

\author{
Azliana Aziz \\ (Corresponding author) \\ MB Bch BAO, MMed ORL-HNS \\ Department of Otorhinolaryngology- \\ Head \& Neck Surgery, School of \\ Medical Sciences, Universiti Sains \\ Malaysia, Kelantan, Malaysia \\ Email: az_aziz@usm.my
}

Department of Otorhinolaryngology-

Head \& Neck Surgery, Hospital

Universiti Sains Malaysia, 16150

Kubang Kerian, Kelantan, Malaysia

\section{Nur Saadah Mohamad}

MD

Department of OtorhinolaryngologyHead \& Neck Surgery, School of Medical Sciences, Universiti Sains Malaysia, Kelantan, Malaysia

Department of OtorhinolaryngologyHead \& Neck Surgery, Hospital Universiti Sains Malaysia, 16150 Kubang Kerian, Kelantan, Malaysia

Mohd Jazman Che Rahim MD, MMed Internal Medicine Internal Medicine Department, School of Medical Sciences, Universiti Sains Malaysia, Kelantan, Malaysia

Department of Internal Medicine Hospital Universiti Sains Malaysia, 16150, Kubang Kerian Kelantan, Malaysia

\begin{abstract}
The clavicle or collarbone is a horizontal-axis bony structure located between the neck and thoracic area. Tumour metastasis at the clavicle is very rare. Due to its location at the border of the neck and chest area, a primary tumour could originate from both areas. We report the case of a 39-year-old man who presented with a painful right sternal-end clavicular mass and intermittent fever. Chest radiography was normal. Musculoskeletal ultrasound of the clavicle revealed a mass. Computed tomography (CT) thorax further identified a mass at the upper lobe of the right lung. CT-guided tissue biopsy confirmed that it was a lung adenocarcinoma. This case shows an atypical presentation of lung carcinoma and how musculoskeletal ultrasound helped in the diagnosis when other features and investigations were inconclusive.
\end{abstract}

\section{Introduction}

Clavicular swelling is most commonly due to trauma or non-union healing fractures. Inflammation and bone tumours are the other possible causes. There are few reported cases of tumours involving the clavicle, which account for only $0.45-1.01 \%$ of all bony tumours. ${ }^{1}$ Most of these clavicular tumours were malignant and composed of Ewing's sarcoma, ${ }^{1}$ osteosarcoma ${ }^{2}$ and myeloma. ${ }^{3}$ Meanwhile, other differential diagnoses include osteomyelitis, tuberculosis and infection at the sternoclavicular joint area. However, lung carcinoma with clavicular metastasis has never been reported despite the proximity of both structures.

\section{Case history}

A 39-year-old man presented to the emergency department with a complaint of pain and progressively enlarged right medial clavicular swelling with intermittent fever over a 4-month duration. He had no recent trauma, fall or traditional massage. He also had no cough, dyspnoea, haemoptysis, loss of weight, dysphagia, odynophagia or noisy breathing. He was an active smoker of 25 pack-years. Since he was a construction worker, he was constantly exposed to cement and concrete dust. There was no history of head or neck radiation, contact with tuberculosis patients or malignancy in his family. Although he previously sought treatment at multiple clinics and was given a few courses of oral analgesics and antibiotics, the pain and swelling were not reducing.
On examination, there was a firm-to-hard swelling at the right medial part of the clavicle measuring $3 \times 3 \mathrm{~cm}$. It was immobile and mildly tender on palpation. The overlying skin was normal. There were no palpable neck nodes. Bilateral upper limb neurological findings were normal. On the chest and clavicle radiographs, the right clavicle had a heterogeneously thickened medial end that was suspicious of a soft tissue lesion or lymph node (Figure 1). The lungs and remaining bones appeared normal. Tuberculosis workup (tuberculin skin test and sputum for acid-fast bacilli) were negative. Serum calcium level was normal. He was covered with intravenous augmentin $(1.2 \mathrm{~g})$ thrice a day for one week whilst admitted. However, the swelling and pain were not reducing. The patient's poor response to medical therapy raised the suspicion of malignancy.

Ultrasound of the right sternoclavicular joint was performed to determine the origin and extent of the mass. It revealed a well-defined hypoechoic lesion with cortical destruction at the medial end of the right clavicle, thus raising the suspicion of a tumour or bony metastasis. Computed tomography (CT) neck and thorax showed a spiculated right apical lung mass measuring $4.1 \times 3.4 \times 5$ $\mathrm{cm}$ (Figure 2). There were also bilateral supraclavicular and mediastinal lymph node enlargements, a right adrenal lesion and lytic lesions of the posterior part of the second right rib that were suggestive of distant metastases. CT-guided biopsy of the right apical mass identified it as primary lung adenocarcinoma. Our patient was diagnosed 
with lung adenocarcinoma (stage $\mathrm{T} 1 \mathrm{cN} 3 \mathrm{M} 1$ ). Epidermal growth factor receptor (EGFR) mutations were negative. The clavicular swelling and pain resolved after two courses of platinumbased chemotherapy.
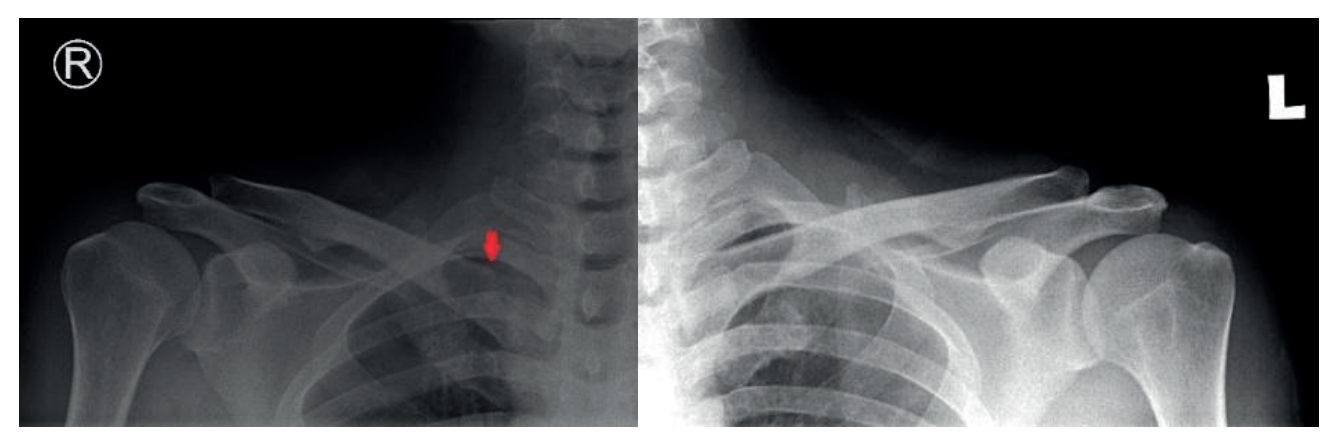

Figure 1. A clavicular radiography study showed an irregular margin of the medial end of the right clavicle when compared to the medial end of the left clavicle (red arrow).
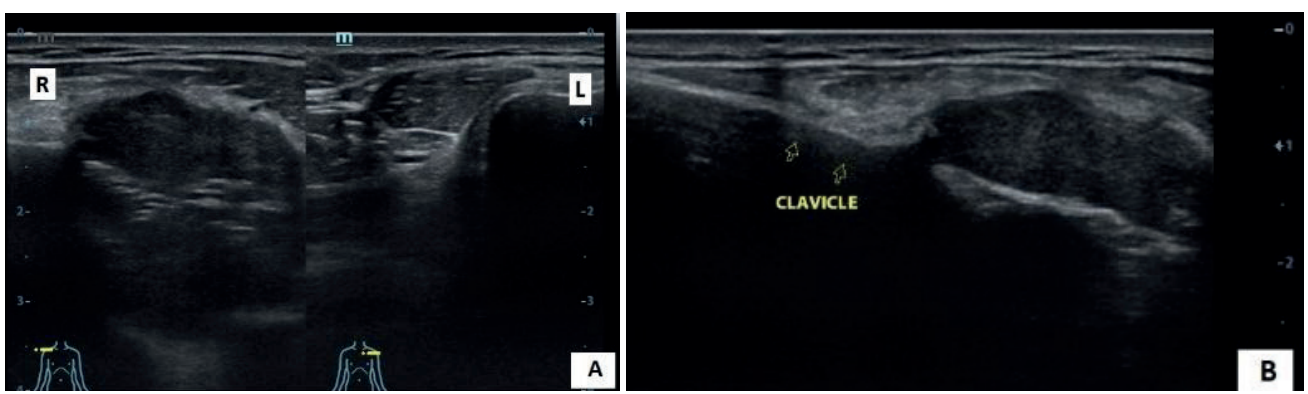

Figure 2. Ultrasound images of the bilateral medial end of the clavicle with enlargement of the right clavicle (A). The lesion is hypoechoic with bony cortical destruction (B).
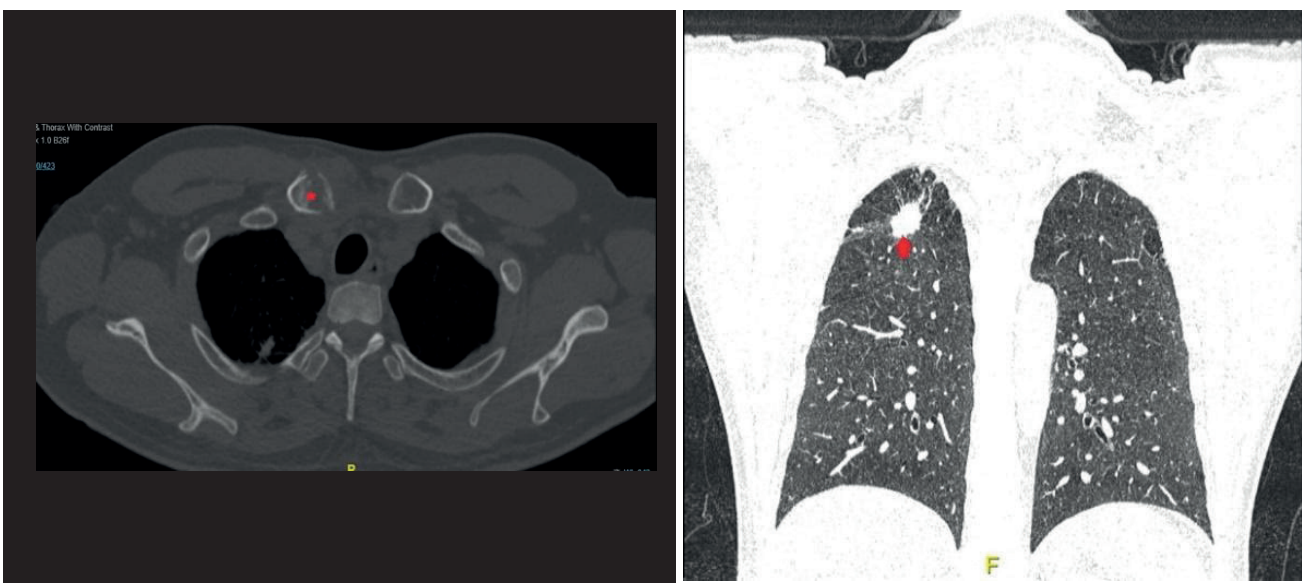

Figure 3. A computed tomography image showing a lytic lesion with an erosion of the bony cortex of the sternal end of the right clavicle (red asterisk, axial view) and a small right apex of lung lesion measuring $1.19 \times 2.56 \mathrm{~cm}$ (red arrow, coronal view).

\section{Discussion}

Trachea, bronchus and lung carcinoma is the second most common group of cancer among males and the fifth most common among females in Malaysia. ${ }^{4}$ Lung adenocarcinoma is the most common lung malignancy and strongly associated with smoking. A study by Noor et al. ${ }^{5}$ showed that chronic exposure to cement dust increased the risk for lung, laryngeal and gastrointestinal cancer as well as dermatitis. Our patient was at risk for lung cancer since he was an active smoker and worked in the construction sector, whereby he was constantly exposed to cement and concrete dust.

It is very rare for lung malignancy cases to present with a painful clavicular mass without any respiratory or constitutional symptoms. It is also rare for lung carcinoma to invade the clavicles since it is more commonly found to metastasise to the vertebrae, pelvis, humerus 
and femur. Clavicles are long flat bones that have no bone marrow. ${ }^{1}$ Cancer tends to spread to long medullary bones since there is more blood supply and the bone marrow provides a fertile microenvironment for the metastatic cancer cells to flourish. ${ }^{6}$ In our case, the tumour had most likely spread haematogenously since there was no direct extension from the right apical lung mass to the clavicle based on the CT images.

The clavicular swelling was hard in consistency with no egg-shell crackling sensation on palpation. Egg-shell crackling is a crepitus sensation during the palpation of a bony mass due to thinning of the bony cortex by the tumour mass. The likely reason for this is that the erosion of the bony cortex was at the medial side of the bone, which is a limited area for assessment. A radiography study is an easily available investigation that can be used to assess bony abnormalities. It has high specificity in detecting lesion features, which may help in determining the locations of lesions and their biological activity. ${ }^{7}$ However, it has a limited ability to visualise lesions near complex structures (e.g. ribs, the sternum, vertebrae), which require other modalities such as CT or Magnetic Resonance Imaging (MRI). In our case, a sternoclavicular joint ultrasound was able to detect the lytic lesion at the medial part of the sternal-end clavicle that was difficult to interpret in the radiography study. The lung mass was also obscured by the bony shadow.

The clavicle is a subcutaneously located bone. Thus, any lumps should be detected early. Despite its superficial location, tissue biopsy methods must be chosen wisely, especially at the medial part of the clavicle due to close proximity to vital structures such as blood vessels, nerve bundles, lungs and the trachea. A needle biopsy poses the risk of injuring the adjacent neurovascular structure and causing pneumothorax. ${ }^{1}$ There is also a risk of pathological fracture since bone consistency can be softened due to tumour erosion. Thus, hard manipulation during core biopsy should also be avoided. An open biopsy provides a surgical view that helps in targeting the tissue, acquiring a larger sample and avoiding injuries to nearby vital structures. The chosen biopsy method should strike a balance between the highest yield of tissue and minimising procedural complications. Fortunately, in our case, a CT-guided tissue biopsy of the lung mass was able to achieve the tissue diagnosis, thus avoiding the aforementioned complications of bone biopsy.

\section{Conclusion}

Persistent bone pain and swelling that do not respond to medical treatment should raise suspicions of malignancy. Nontraumatic painful clavicular swelling is rare and a high degree of suspicion is required for early diagnosis and treatment. Moreover, multidisciplinary team management is required with the integration of clinical, radiological and pathological inputs for a definite diagnosis, especially in such rare cases.

\section{Acknowledgement}

We would like to thank the patient who gave us permission for the publication of this case report. Moreover, we also thank all of the teams involved in treating this patient.

\section{Conflicts of interest}

All authors declare no conflicts of interest.

Patient's consent for the use of images and content for publication

Verbal consent obtained from the patient for the use of images and his case for the publication of this case report.

\section{What is the implication to patients?}

The awareness of the potential of clavicular metastasis in lung malignancy by the health care professionals is important, as it can cause a delay in early referral to the tertiary centre for appropriate investigations to establish the definitive diagnosis and subsequently delay in initiating treatment. 


\section{References}

1. Kapoor S, Tiwari A, Kapoor S. Primary tumours and tumorous lesions of clavicle. Int Orthop. 2008;32(6):829.

2. Smith J, Yuppa F, Watson RC. Primary tumors and tumor-like lesions of the clavicle. Skeletal Radiol. 1988;17(4):235-46.

3. Pratt GF, Dahlin D, Ghormley R. Tumors of the scapula and clavicle. Surg Gynecol Obstet. 1958;106(5):536-44.
4. Azizah AM, Nor Saleha I, Noor Hashimah A, et al. Malaysian National Cancer Registry Report 2007-2011. Malaysia Cancer Statistics, Data and Figures. Putrajaya: National Cancer Institute, Ministry of Health; 2016.

5. Noor H, Yap C, Zolkepli O, et al. Effect of exposure to dust on lung function of cement factory workers. Med J Malaysia. 2000;55(1):51-7.
6. Berenson J, Rajdev L, Broder M. Pathophysiology of bone metastases. Cancer Biol Ther. 2006;5(9):1078-81.

7. Priolo F, Cerase A. The current role of radiography in the assessment of skeletal tumors and tumor-like lesions. Eur J Radiol. 1998; 27:77-85. 